\title{
MODIFICACIÓN AL PROCESO DE PRODUCCIÓN MASIVA DE LOS PARASITOIDES Catolaccus grandis Y Catolaccus hunteri ${ }^{1}$
}

\author{
Marco Antonio Reyes-Rosas ${ }^{2}$, Jesús Loera-Gallardo², José Isabel López-Arroyo ${ }^{3}$
}

\section{RESUMEN}

Modificación al proceso de producción masiva de los parasitoides Catolaccus grandis y Catolaccus hunteri. El presente estudio se realizó con el objetivo de modificar la tecnología de producción masiva de Catolaccus grandis y Catolaccus hunteri, himenópteros parasitoides de Anthonomus grandis el picudo del algodonero en Tamaulipas, México en el 2002. Se determinó la eficiencia del papel toalla para el secado de manos, en el sellamiento de cápsulas moldeadas en cera de hidrocarburos y poliolefina (LCHP), donde se confina al huésped, y evaluar la efectividad del gorgojo del garbanzo Callosobruchus maculatus como hospedante facticio para la cría de ambos parasitoides. Hubo producción masiva de $C$. grandis y $C$. hunteri utilizando $C$. maculatus como huésped y el papel toalla fue efectivo para sellar los orificios de las cápsulas moldeadas en LCHP. La producción masiva de $C$. grandis y $C$. hunteri mediante $C$. maculatus, contribuyó a incrementar la seguridad y facilidad del proceso, además de una significativa reducción de costos. El papel toalla en el sellado de los orificios de las cápsulas moldeadas en LCHP, redujo en un 50\% por $\mathrm{m}^{2}$ los costos del proceso, sin afectar la eficiencia del parasitismo y emergencia de $C$. grandis.

Palabras clave: Encapsulación artificial, Callosobruchus maculatus, huésped facticio, algodonero, insectos benéficos.

\begin{abstract}
Modifications to the process of mass production of parasitoids Catolaccus grandis and Catolaccus hunteri. The present study was conducted to modify the actual mass rearing technology for Catolaccus grandis and Catolaccus hunteri, hymenoptera parasitoids of Anthonomus grandis, the cotton boll weevil, in Tamaulipas, México during 2002. The efficiency of the paper towel to seal the wax hydrocarbon polyolefin molded capsules where the host is confined was determined; and the Chickpea weevil Callosobruchus maculatus effectivity was evaluated as a facticious host to rear both parasitoids. The results indicated that $C$. grandis y C. hunteri mass reared through C. maculatus as a facticious host was possible, and paper towel was effective in sealing the holes in molded WHPS capsules. This mass rearing of $C$. grandis y $C$. hunteri by using $C$. maculatus may contribute to increase security and easiness in the process, and a significant reduction in costs. Paper towel used to seal holes in molded WHPS capsules reduce costs in 50\% per square meter of material, without affecting parasitism and emergence of de C. grandis de C. grandis.
\end{abstract}

Key words: Artificial encapsulation, Callosobruchus maculatus, facticious host, cotton plant, beneficial insects.

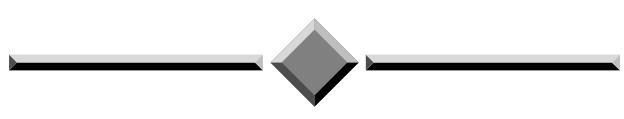

Recibido: 19 de mayo, 2009. Aceptado: 17 de mayo, 2010.

2 Instituto Nacional de Investigaciones Forestales, Agrícolas y Pecuarias, Campo Experimental Río Bravo, Apartado Postal 172 , Km 61 Carretera Matamoros-Reynosa. 88900 Río Bravo, Tamaulipas, México. reyes.marco@inifap.gob.mx, pezmar66@yahoo.com.mx, loera. jesus@inifap.gob.mx

3 Instituto Nacional de Investigaciones Forestales, Agrícolas y Pecuarias (INIFAP), Campo Experimental General Terán. Km. 31 Carretera Montemorelos-China. 67400 General Terán, N.L., México. jila64@yahoo.com.mx 


\section{INTRODUCCIÓN}

El picudo del algodonero, Anthonomus grandis Boheman (Coleoptera: Curculionidae), es uno de los problemas fitosanitarios más importantes y el de mayor impacto en la producción de algodonero en México (Vázquez 1998). El área sembrada con este cultivo ha decrecido notablemente en los últimos años. Las causas son atribuidas a los altos costos de inversión y a los bajos precios de la fibra en el mercado. Para el control de plagas se utiliza hasta el $60 \%$ de los costos de inversión, y en su mayor parte son destinados para el control de A. grandis, el cual llega a ocasionar pérdidas estimadas en un $40 \%$ del rendimiento (Vargas et al. 1998). Como una medida alternativa, se ha considerado el control biológico mediante parasitoides, entre los cuales destacan Catolaccus grandis (Burks), C. hunteri Crawford (Hymenoptera: Pteromalidae) y Bracon mellitor Say (Hymenoptera: Braconidae) como las especies más promisorias (Reyes et al. 2007, Loera et al. 2008).

C. grandis ha mostrado resultados sobresalientes en liberaciones inundativas, contra A. grandis (Summy et al. 1995, King et al. 1995, Vargas et al. 1997). En Río Bravo y Llera, Tamaulipas, México, causó 88\% de mortalidad en larvas de tercer instar y prepupas del picudo del algodonero, incremento de $32 \%$ en rendimiento y reducción de $90 \%$ en las aplicaciones de insecticidas (Vargas et al. 1997, 1998). Para C. hunteri se ha registrado parasitismo en $A$. grandis en un porcentaje similar o mayor que $C$. grandis, cuando ambos se presentaron en condiciones naturales (Cate et al. 1990, Pacheco 1996); en evaluación de campo, C. hunteri y C. grandis, causaron un parasitismo de $40 \%$ y $42 \%$, respectivamente, (Cortez et al. 2004). El potencial de estos parasitoides para reducir las poblaciones de $A$. grandis en algodonero, y su integración a un programa de manejo, es considerado relevante para reducir costos y uso de insecticidas, fundamentando la necesidad de dar continuidad al establecimiento de un programa de cría masiva y liberación, de ambos parasitoides.

Cate (1987) desarrolló un método de reproducción masiva, mediante larvas de A. grandis como huésped, para exponerlas al parasitoide, encapsuladas entre dos láminas de cera de hidrocarburos y poliolefina (LCHP), material muy eficiente pero costoso. Este proceso fue utilizado en el ARS-USDA en Weslaco, Texas, EUA y en el INIFAP en Río Bravo, Tamaulipas, México, pero los elevados costos en su encapsulación impidieron el seguimiento de esta actividad. Sin embargo, es posible que la segunda LCHP, utilizada para sellado, pueda ser reemplazada con un material más económico pero con igual eficiencia.

Por otra parte, la reproducción del huésped natural A.grandis resulta difícil en ausencia de su dieta natural; y aunque se han logrado avances en el desarrollo de una dieta artificial (Rojas et al. 1996), los resultados no han sido alentadores; es de alto costo, y frecuentemente se contamina por hongos y bacterias, ocasionando pérdidas y retraso en la producción. El reemplazo de A. grandis por un huésped facticio (huésped no natural pero que permite el desarrollo del parasitoide) ofrece una posibilidad para reducir costos y facilitar la producción masiva de $C$. grandis y $C$. hunteri en ausencia del huésped natural, sin detrimento en su capacidad reproductiva (Bárcenas et al. 1997).

El objetivo de este trabajo, fue modificar la técnica de producción masiva de $C$. grandis y $C$. hunteri, con materiales más económicos.

\section{MATERIALES Y MÉTODOS}

El estudio fue realizado en el año 2002, en el Campo Experimental Río Bravo (CERIB), Tamaulipas, México ( $25^{\circ} 57^{\prime} 53^{\prime}$ ' N, $98^{\circ} 01$ '03,3” O), del Instituto Nacional de Investigaciones Forestales, Agrícolas y Pecuarias (INIFAP).

\section{Adaptabilidad de parasitoides al huésped facticio C. maculatus}

Se utilizó C. maculatus como huésped facticio, y como testigo para comparación al huésped natural A. grandis; ambos fueron expuestos a ser parasitados por $C$. grandis y $C$. hunteri. C. grandis fue producido en $C$. maculatus durante 13 generaciones, excepto la quinta generación que fue criada en A. grandis; mientras que $C$. hunteri fue reproducido exclusivamente en C. maculatus durante 50 generaciones.

De cada especie parasitoide se seleccionaron al azar 50 hembras y 25 machos recién emergidos y se mantuvieron en condiciones de $27^{\circ} \mathrm{C}, 70 \%$ humedad relativa (HR) y 12:12 luz:oscuridad (L:O) h, en una jaula de acrílico transparente de 40x40x40 cm, alimentados con miel de abeja dispuesta en bandas de cartón colocadas en la parte superior del recipiente, y agua, disponible mediante un mechón de fibra de algodón 
colocado en vasos de $30 \mathrm{ml}$. Durante los primeros dos días se les administró larvas de $A$. grandis encapsulado artificialmente, para inducir la oviposición (Morales 1998). Al tercer día de edad, se introdujeron 10 hembras y cinco machos de cada especie parasitoide, por separado, en cada uno de cinco recipientes de plástico de 3,5 1, cubriendo el orificio de salida con tela de poro de 250 micras. En cada recipiente de plástico se introdujeron dos láminas de parasitación (Cate 1987) cada una con 64 cápsulas. En la lámina de A. grandis se introdujo una larva por cápsula y dos en la de $C$. $m a$ culatus por su menor tamaño, para simular la biomasa de una larva de picudo de tercer instar (Figura 1). Los huéspedes fueron expuestos al parasitoide durante 24 $\mathrm{h}$, posteriormente, las láminas fueron reemplazadas con nuevos huéspedes de la misma edad. Diariamente se registró la mortalidad de ambos parasitoides, así como el número de huevos ovipositados en cada huésped. Los huevos fueron detectados y contabilizados a contra luz mediante una lámpara de luz blanca. Los datos fueron analizados como grupos apareados mediante una prueba de $t$ de Student $(\alpha=0,05)$ (SAS 1988).

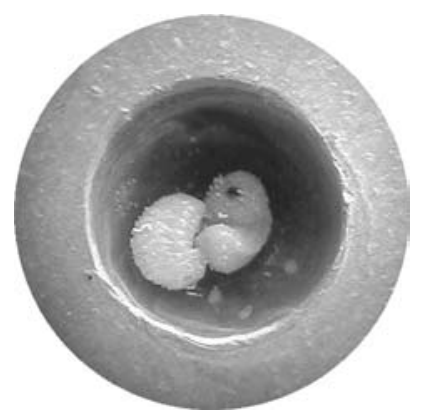

Figura 1. Larvas de quinto estadío del gorgojo del garbanzo Callosobruchus maculatus. Río Bravo, Tamaulipas, México. 2002.

Eficiencia del papel toalla en la encapsulación del huésped

Las cápsulas o burbujas para encapsular al huésped fueron moldeadas en LCHP de $20 \times 10 \mathrm{~cm}$, material que se caracteriza por estirar hasta diez veces su talla antes de romperse. En cada lámina se moldearon 126 cápsulas y los orificios fueron sellados con LCHP, las dos láminas se unieron al ejercer presión (Cate
1987) o con papel toalla común para secado de manos, uniéndolo a la lámina con cápsulas de LCHP, de la misma manera. En cada cápsula se confinaron dos larvas del huésped. Las láminas se colocaron en forma de cilindro, con las cápsulas hacia la cara exterior (Figura 2).

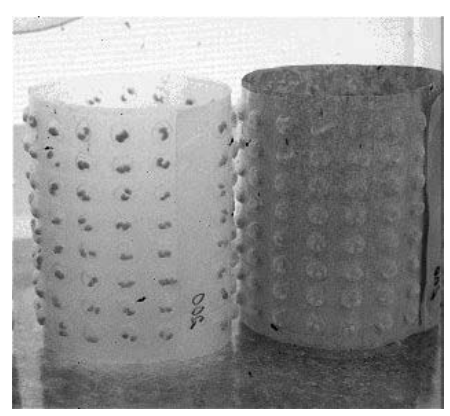

Figura 2. C. maculatus encapsulado en LCHP-LCHP (izquierda) y LCHP-papel (derecha). Río Bravo, Tamaulipas, México. 2002.

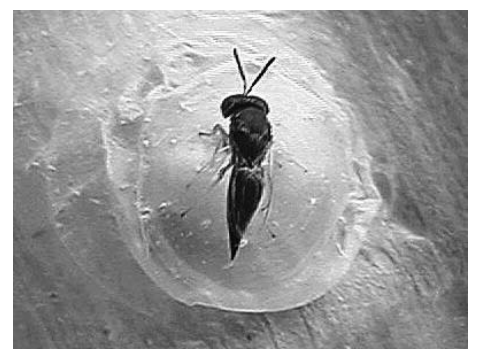

Figura 3. C. grandis ovipositando sobre larvas de C. maculatus encapsuladas artificialmente. Río Bravo, Tamaulipas, México. 2002.

Como parasitoide se utilizó a $C$. grandis adulto (Figura 3), de tres días de edad y como huésped a larvas de $C$. maculatus en su cuarto o quinto instar.

Se seleccionaron al azar 250 hembras y 25 machos del parasitoide $C$. grandis (Figura 3), recién emergidos; se alimentaron con miel de abeja y agua en proporción 20:80 y se mantuvieron en condiciones de $27^{\circ} \mathrm{C}, 65 \pm 5 \%$ H. R. y 15:9 L:O, en una jaula de acrílico transparente de 40x40x40 cm. Previo al ensayo, larvas del huésped, encapsuladas, fueron expuestas al parasitoide con el propósito de inducir la oogénesis (Morales 1998). 
Los tratamientos fueron: 1) C. maculatus encapsulado en LCHP y sellado con LCHP y 2) C. maculatus encapsulado en LCHP y sellado con papel toalla. Durante los primeros cuatro días se introdujeron a la jaula de acrílico dos láminas de encapsulación con el tratamiento uno y dos, y durante los 11 días subsecuentes, se introdujeron diariamente seis láminas en cada tratamiento. Cada $24 \mathrm{~h}$, las láminas fueron reemplazadas por nuevo material y se colocaron en cajas de acrílico similares a la descrita previamente; a los 15 días después se registró el número de $C$. grandis emergidos. Los datos fueron analizados como grupos apareados mediante una prueba de $\mathrm{t}$ de Student $(\alpha=0,05)($ SAS v 6,12).

\section{RESULTADOS Y DISCUSIÓN}

\section{Adaptabilidad de parasitoides al huésped facticio C. maculatus}

Adaptabilidad de $C$. grandis. Durante los primeros seis días las oviposturas de $C$. grandis fueron mayores en $C$. maculatus y a partir del séptimo día y hasta el fin del ensayo, las oviposturas fueron mayores en $A$. grandis, entre el $64 \%$ y $82 \%$.

A partir del onceavo día, el número de oviposturas del parasitoide disminuyeron en ambos huéspedes pero el decremento fue más notable en $C$. maculatus. La mayor cantidad de oviposturas ocurrida en A. grandis fue de 496 el día diez y en C. maculatus fue de 281 el día cuatro (Figura 4).

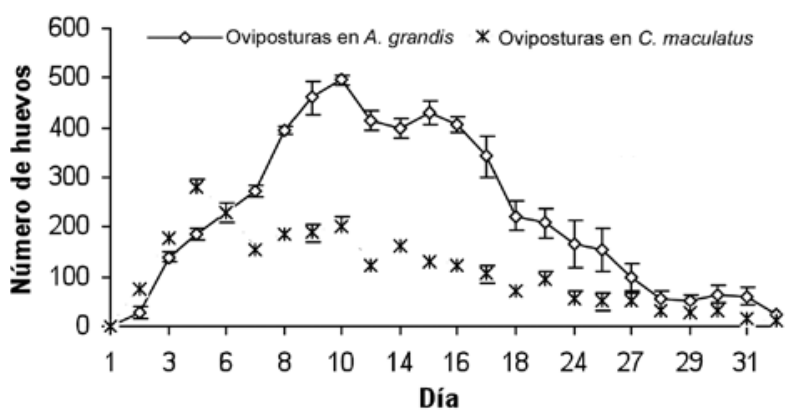

Figura 4. Oviposturas de Catolaccus grandis en larvas del huésped natural Anthonomus grandis y en el huésped facticio Callosobruchus maculatus encapsulados artificialmente. Río Bravo, Tamaulipas, México. 2002.
Durante el periodo del ensayo, la población total de huevos de $C$. grandis en ambos hospedantes fue de 7868 , el 67,3 y $32,7 \%$ de las oviposturas se dio en $A$. grandis y $C$. maculatus, respectivamente, lo que indicó una clara preferencia por su huésped natural. Rojas et al. (1999) reportan que la cría de diez generaciones de $C$. grandis en el huésped facticio $C$. maculatus reduce su aceptación de $A$. grandis como huésped, sin embargo, en el presente estudio $C$. grandis fue criado en $C$. maculatus por más de diez generaciones y el periodo y cantidad de oviposturas fue similar a las que se han obtenido en producciones masivas que utilizan A. grandis para la reproducción de $C$. grandis.

La mortalidad del parasitoide $C$. grandis fue de $50 \%$ al día 18 y, el día 38 se murió el último individuo.

La proporción de hembras de $C$. grandis obtenida del huésped A. grandis fue mayor que la obtenida en el huésped C. maculatus, correspondiendo a 4:1 y 2:1 respectivamente. Esta diferencia pudiera relacionarse con el tamaño del huésped facticio que es más apropiado para el desarrollo del macho. Algunas avispas parasíticas depositan huevos que producirán machos en huéspedes pequeños y viceversa, así mismo la mortalidad en hembras es mayor que en machos cuando el tamaño del huésped es menor al adecuado para su desarrollo (Kishi 1970, Sandlan 1979, Charnov 1892, King 1987). El comportamiento para la determinación del sexo en $C$. grandis en función al tamaño del hospedero no se ha establecido.

La relación hembra-macho obtenida en el presente estudio fue inferior a la observada a la producción masiva rutinaria de $C$. grandis en Río Bravo, Tamaulipas, donde se obtuvo una proporción hembra-macho de 5:1, utilizando A. grandis como huésped. Coleman $(2009)^{4}$ señala que en Weslaco Texas, la proporción obtenida es 6:1 con el mismo huésped, aunque esta proporción puede variar por diversos factores. Cabe señalar que en ambas crías masivas, el parasitoide $C$. grandis, nunca estuvo expuesto a los dos huéspedes al mismo tiempo, por lo que esta condición pudo influir en el resultado de la relación hembra-macho.

Adaptabilidad de $\boldsymbol{C}$. hunteri. Las oviposturas de $C$. hunteri se incrementaron notablemente el tercer día en ambos huéspedes (Figura 5). Sin embargo, las oviposturas ocurrieron consistentemente en proporciones mayores sobre A. grandis desde el primero y hasta

\footnotetext{
${ }^{4}$ Coleman, R. 2009. Producción masiva de Catolaccus grandis (entrevista). ARS-United State Department of Agriculture (USDA), Lab. Biological Control, Weslaco Texas. USA.
} 


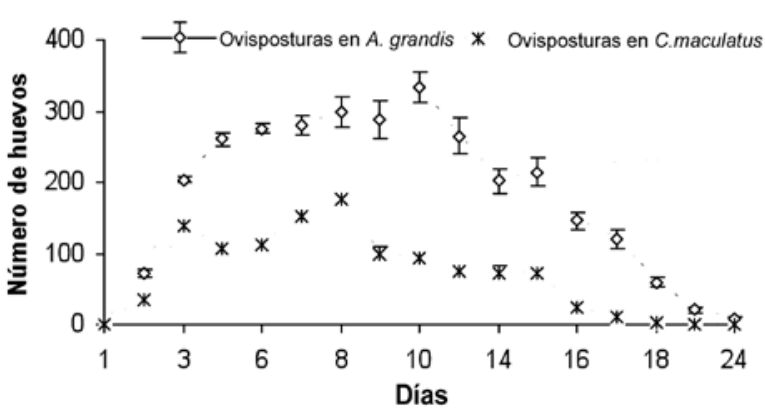

Figura 5. Oviposturas de Catalaccus hunteri en larvas del huésped natural Anthonomus grandis y en el huésped facticio Callosobruchus maculatus, encapsuladas articialmente. Río Bravo, Tamaulipas, México. 2002.

el último día del ensayo, dentro de un rango del 60 al $100 \%$. C. hunteri mostró una marcada preferencia por A. grandis, a pesar de que fue criado en el huésped facticio C. maculatus durante 50 generaciones. Entre los días 10 y 11, se inició la disminución de oviposturas en C. maculatus y A. grandis, respectivamente, aunque la tendencia de preferir a. A. grandis se mantuvo constante (Figura 5).

La mayor cantidad de oviposturas ocurrida en $A$. grandis fue de 333 el día diez y en $C$. maculatus fue de 175 el día ocho.

Durante el periodo del ensayo, la población total de huevos de $C$. hunteri en ambos hospedantes fue de 4221 , correspondiendo a 72,2 y $27,8 \%$ de oviposturas en $A$. grandis y $C$. maculatus, respectivamente. Al día 16 la mortalidad registrada de C. grandis fue de 56\% y el día 27 se registró la mortalidad del último individuo. La proporción de sexos de C. hunteri fue de 3:1 hembras:macho en ambos hospederos. No se apreció influencia del huésped en la proporción sexual, como la observada en $C$. grandis; posiblemente a la semejanza en biomasa de las larvas de $C$. maculatus con la del picudo del chile Anthonomus eugenii uno de los hospederos frecuentemente asociado a este parasitoide (Rodríguez y Reyes 2003, Rodríguez et al. 2007, Cortez 2008).

De acuerdo a los resultados obtenidos, la reproducción de $C$. grandis y de $C$. hunteri por más de diez generaciones en el huésped facticio $C$. maculatus, no afectó su preferencia de parasitismo por A. grandis, por lo que la producción masiva de $C$. grandis y $C$. hunteri utilizando $C$. maculatus como huésped facticio, puede contribuir a incrementar la seguridad y facilidad de la producción de estos parasitoides en ausencia de su huésped natural, lo que abre la posibilidad de su incorporación a programas de control biológico de plagas como la del picudo del chile A. eugenii y picudo del algodonero A. grandis.

\section{Eficiencia del papel toalla en la encapsulación del huésped}

En nueve de los quince días del ensayo la emergencia de $C$. grandis en el tratamiento LCHP - papel toalla fue mayor que en el tratamiento LCHP-LCHP (Figura 6).

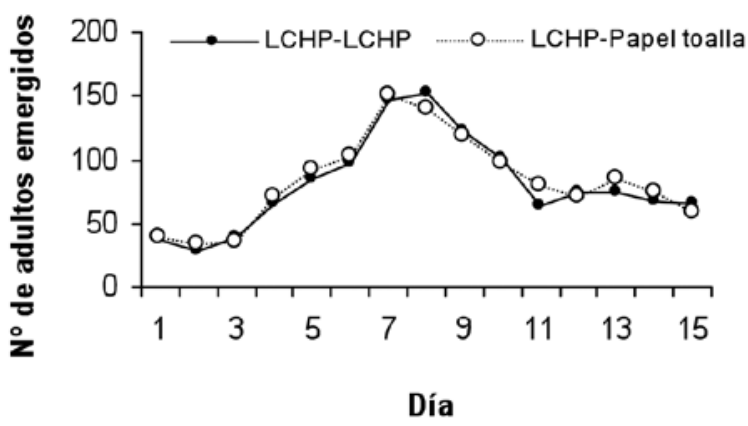

Figura 6. Número de adultos de Catalaccus grandis emergidos en los tratamientos LCHP-LCHP y LCHP-papel toalla. Río Bravo, Tamaulipas, México. 2002.

No se registraron diferencias significativas en el número de $C$. grandis obtenidos en ambos tratamientos. $C$. grandis oviposita a través de las cápsulas $\mathrm{y}$ estos tratamientos fueron moldeadas en LCHP. Generalmente, las hembras de $C$. grandis producen el mayor número de progenie entre los ocho y quince días de edad (Morales 1998); en el presente estudio su progenie representó el 58 y $59 \%$ en los tratamientos LCHP-papel toalla y LCHP-LCHP, respectivamente, desde el octavo al catorceavo día. La nueva lámina de encapsulación compuesta por LCHP-papel toalla redujo a la mitad el costo de encapsulación por la substitución de una lámina de LCHP por papel toalla, cuyo costo por $\mathrm{m}^{2}$ es de 0,07 dólar. Además de esta ventaja económica, su adhesión a la lámina de LCHP es consistente y no afecta el comportamiento de parasitación 
y emergencia de $C$. grandis. Fue común observar los orificios de salida del parasitoide en ambas caras de las láminas en los dos tratamientos.

Estos resultados confirman la factibilidad de la reproducción masiva de $C$. grandis y $C$. hunteri con materiales más económicos como el papel, el cual podría en un futuro sustituir completamente al LCHP.

\section{AGRADECIMIENTOS}

Los autores agradecen al Instituto Nacional de Investigaciones Forestales, Agrícolas y Pecuarias (INIFAP) México y al Departamento de Agricultura de los Estados Unidos A.R.S.-U.S.D.A., por el otorgamiento de fondos para el proyecto "Control biológico del picudo del algodonero mediante el uso de la avispita Catolaccus grandis", sin los cuales no hubiera sido posible la realización de esta investigación.

\section{LITERATURA CITADA}

Bárcenas, ON; Meraz, AR; Aquino, PG; King, EG. 1997. Cría de Catolaccus grandis (Burks) (Hymenoptera: Pteromalidae) en el huésped facticio Callosobruchus maculatus F. (Coleoptera: Bruchidae): Efecto del número de huésped por celda. In: Memorias del Congreso Nacional de Control Biológico. Sociedad Mexicana de Control Biológico. Guadalajara, Jal. México. p. 184-186.

Cate, JR 1987. A method of rearing parasitoids of boll weevil without the host plant. Southwestern Entomologist 12:211-215.

Cate, JR; Krauter, PC; Godfrey, KE. 1990. Pest of cotton, In: DH Habeck, FD Bennet, JH Frank. ed. Classical Biological in the Southern United States. South. Coop. Ser. Bull. 355. p. 17-29.

Charnov, EL. 1982. The theory of sex allocation. Princeton University Press, Princeton, NJ. 378 p.

Cortez, ME; Bárcenas, ON; Martínez JL; Leyva J.L; Vargas J y Rodríguez del Bosque LA. 2004. Parasitismo de Catolaccus grandis y Catolaccus hunteri (Hymenoptera: Pteromalidae) sobre el picudo del algodonero Anthonomus grandis Boheman (Coleoptera: Curculionidae). Agrociencia 38:497.

Cortez-Mondaca, E. 2008. Picudo del chile Anthonomus eugenii (Coleoptera:Curculionidae). In: Arredondo, BHC; Rodríguez, del BLA. eds. Casos de control biológico en México. p. 127-136
King, BH. 1987. Offspring sex rations in parasitoid wasps. Quarterly Review of Biology 62:367-396.

King, EG; Coleman, J; Woods, L; Wendel, L; Greenberg, S; Scott, AW. 1995. Suppression of the boll weevil in commercial cotton by augmentative releases of the wasp parasite, Catolaccus grandis. In: Addendum to the Proceedings Beltwide Cotton Conferences 1995, National Cotton Council of America, Memphis, TN. p. 26-30.

Kishi, Y. 1970. Difference in the sex ratio of the pine bark weevil parasite, Dolichomitus sp. (Hymenoptera: Ichneumonidae), emerging from different host species. Applied Entomology and Zoology 5:126-132.

Loera-Gallardo, J; Reyes-Rosas, M; López-Arroyo, JI. 2008. Picudo del algodonero Anthonomus grandis (Coleoptera:Curculionidae). In: Arredondo, BHC; Rodríguez, del BLA. eds. Casos de control biológico en México. p. $75-87$.

Morales-Ramos, JA. 1998. Biología y ecología de Catolaccus grandis (Hymenoptera: Pteromalidae). Vedalia 5 (1):97-109.

Pacheco, MF. 1996. Plagas de los cultivos oleaginosos en México. Campo Experimental Valle del Yaqui, SARH-INIFAP-CIRNO. Libro Técnico. Cd. Obregón, Sonora, México. 414 p.

Reyes-Rosas, MA; Loera-Gallardo J; López Arroyo JI; Vargas-Camplis, J. 2007. Parasitoides hymenópteros de Anthonomus grandis (Coleoptera: Curculionidae) en el Norte de Tamaulipas. Southwestern Entomologist 32(1):53-64.

Rodríguez-del-Bosque, LA; Reyes-Rosas, MA. 2003. Damage, survival and parasitism, of Anthonomus eugenii (Coleoptera: Curculionidae) on piquin pepper in northern Mexico. Southwestern Entomologist 28(4):293-294.

Rodríguez-Leyva, E; Stansly, PA; Schuster DJ; Bravo, ME. 2007. Diversity and distribution of parasitoids of $\mathrm{An}$ thonomus eugenii (Coleoptera: Curculionidae) from Mexico and prospects for biological control, Florida Entomologist 90(4):693-702.

Rojas, MG; Morales, JA; King, EG. 1996. In vitro rearing of the boll weevil (Coleoptera: Curculionidae) ectoparasitoid Catolaccus grandis (Hymenoptera: Pteromalidae) on a meridic diet. J. Econ. Entomol. 89:1095-1104.

Rojas, MG; Morales-Ramos, JA; King, EG. 1999. Response of Catolaccus grandis (Hymenoptera: Pteromalidae) to its natural host after ten generations of rearing on a factitious host, Callosobruchus maculatus. Environ Entomol. 25:137-141.

Sandlan, K. 1979. Sex ratio regulation in Coccygomimus turionellae Linnaeus (Hymenoptera: Ichneumonidae) 
and its ecological implications. Ecological Entomology 4:365-378.

SAS Institute, Inc. 1988. SAS Use's Guide: Statics. Release 6.03. ed. SAS Institute, Inc. Cary, NC. 1208 p.

Summy, KR; Morales-Ramos, JA; King, E.G. 1995 Suppression of boll weevil infestation of South Texas cotton by augmentative releases of the exotic parasite Catolaccus grandis (Hymenoptera: Pteromalidae). Biological Control 5:523-529.

Vargas, C J; Coleman RJ; González J; Rodríguez del Bosque L. 1997. Life table analysis of cotton boll weevil in the tropics of Tamaulipas Mexico after Catolaccus grandis releases. In: Proceedings Beltwide Cotton Conferences. National Cotton Council of America, Memphis, TN, USA. p. 1194-1197.

Vargas, CJ; Coleman RJ; Rodríguez del B, L. 1998. Control del picudo del algodonero en México mediante liberaciones aumentativas de Catolaccus grandis (Himenoptera: Pteromalidae). Vedalia 5:117-122.

Vázquez, JN. 1998. Breve historia del picudo del algodonero en México y de los métodos empleados para su control. Vedalia 5(1):61-70. 